\title{
Research on Grid Scroll Chaotic Extended Sequence Algorithm and Its Circuit Implementation for F-OFDM System
}

\author{
Yingjie Ma, ${ }^{1}$ Ya Li $\mathbb{D},{ }^{2}$ Geng Zhao, ${ }^{1}$ Ping Zeng, ${ }^{1}$ and Yatao Yang ${ }^{1}$ \\ ${ }^{1}$ Beijing Electronic Science and Technology Institute, Beijing, CO 100070, China \\ ${ }^{2}$ Guangdong Polytechnic Normal University, Guangdong, CO 510665, China \\ Correspondence should be addressed to Ya Li; liya2829@gmail.com
}

Received 6 September 2019; Revised 15 October 2019; Accepted 1 November 2019; Published 28 November 2019

Guest Editor: Serdar Çiçek

Copyright (c) 2019 Yingjie Ma et al. This is an open access article distributed under the Creative Commons Attribution License, which permits unrestricted use, distribution, and reproduction in any medium, provided the original work is properly cited.

\begin{abstract}
High peak-to-average power ratio (PAPR), a main problem of 5G Filtered-Orthogonal Frequency Division Multiplexing (FOFDM) system, seriously affects the system's transmission efficiency. Aiming at the shortcomings of the traditional selective mapping (SLM) algorithm, the grid scroll chaotic extended sequence (GSCES) algorithm is proposed to solve the high PAPR problem. The proposed grid multiscroll chaotic maps are used as random phase sequence vectors instead of the traditional pseudo random sequence, which provide a large number of random sequences with good correlation characteristics, random characteristics, and high security. The proposed random phase sequence vectors can meet the needs of $5 \mathrm{G}$ mass connections. The original input signals are divided into real part and imaginary part, which are, respectively, dot produced with the proposed grid multiscroll chaotic maps. The numerical simulation results show that the proposed GSCES algorithm effectively increases the number of candidate sequences, reduces the PAPR values, and improves the transmission efficiency and security. Moreover, the hardware experimental results produce at $2 \times 2 \times 2$-grid multiscroll chaotic attractor, which further proves the physical realization of the proposed system. The proposed schemes have a broad application prospect in $5 \mathrm{G}$ multicarrier modulation technology.
\end{abstract}

\section{Introduction}

In 2015, Filtered-OFDM (F-OFDM) technology was first put forward publicly at the World Mobile Communication Congress. It is an adaptive airport waveform modulation technology whose subcarrier bandwidth can be adjusted according to specific application scenarios. F-OFDM technology has many advantages, such as no need for strict synchronization, reduced out-of-band energy leakage, the different waveform modulation, multiple access technology and frame structure based on the mobile communication application scene and business requirements, the dynamic soft interface parameter configuration according to the $5 \mathrm{G}$ business needs, and packet traffic transmission suitable for Internet of things [1-5]. In the F-OFDM system, when subcarriers of sub-band are superimposed after IFFT operation, multiple subcarriers will reach the peak value at the same time, resulting in higher peak-to-average power ratio
(PAPR) of synthetic signal, which seriously affects the system performance and limits its application in 5G.

The characteristics of selective mapping (SLM) scrambling technology are simple structure and obvious effect, but there are a large side band information and high computational complexity. Many researchers have improved the traditional SLM algorithm. The cyclic-SLM scheme is proposed to omit the side information (SI) and apply only one IFFT. In the time domain, the original signal and its periodic shift signal are added and the minimum PAPR is selected as the candidate signal [6]. The time-frequency domain encryption technique is proposed based on the multichaos and SLM PAPR suppression algorithm. The Lozi and Logistic maps are used to interfere the carrier in the time-frequency domain. In a 100 kilometer single-mode fiber, $8.9 \mathrm{~Gb} / \mathrm{s}$ encrypted OFDM signal is transmitted safely [7]. A method is proposed to decrease the PAPR of the 16-QAM OFDM system, which combines the chaotic map and SLM algorithm 
so that the phase rotation factors are controlled by chaotic sequence [8]. The improved SLM algorithm based on the Lehmer random phase sequence vector is proposed to embed side band information into the 16-PSK transmit signal [9]. The improved SLM algorithm is proposed to reduce PAPR, combining interlaced IDCT transform with pulse forming technology [10]. The low complexity improved SLM algorithm and $\mu$-law compression scheme are proposed to reduce the PAPR and computational complexity of the OFDMA system [11]. The low-complexity SLM improvement algorithm without SI is proposed to reduce the PAPR of the coherent light OFDM system [12]. The blind differential improved SLM algorithm is proposed to reduce the PAPR of Alamouti DSFBC-OFDM system [13].

In this paper, a model of the new system is constructed to produce gird multiscroll chaotic attractors, using different sign functions acted as nonlinear function. Moreover, the design of the hardware circuit produces at $2 \times 2 \times 2$-grid multiscroll hardware experimental results. The produced grid multiscroll chaotic maps are used as random phase sequence vectors instead of the traditional pseudo random sequence. The grid scroll chaotic extended sequence (GSCES) algorithm is proposed to solve the high PAPR problem. The numerical simulation results show that the proposed GSCES algorithm effectively increases the numbers of candidate sequences, reduces the PAPR values, and improves the transmission efficiency and security.

\section{Methods}

2.1. F-OFDM System Modes. F-OFDM system dynamically configures suitable waveform parameters such as bandwidth, cycle prefix (CP) length, subcarrier spacing, and FFT points for each sub-band according to service requirements, which improves the flexibility and scalability of $5 \mathrm{G}$ communication system. The downlink of the F-OFDM system is shown in Figure 1.

As shown in Figure 1, the frequency bands of the system are divided into $M$ sub-bands, which are subcarrier mapping, IFFT transformation, and plus CP operation, respectively, and each sub-band is filtered through the subband filter for coupling transmission. The biggest difference between the F-OFDM system and traditional OFDM system is the sub-band filter added at the transmitter and receiver. For each sub-band, at the transmitter, the input signal of the sub-band filter is the traditional OFDM signal. The function of the sub-band filter is to complete the baseband shaping of each sub-band, and its output signal is the F-OFDM signal. At the receiving end, the function of sub-band filter is to filter each sub-band signal and complete sub-band decoupling.

The data symbol of the $i$ th sub-band of the F-OFDM system is given by

$$
x_{i}(n)=g_{i}(n) * f_{i}(n), \quad i=1,2, \ldots, M,
$$

where $g_{i}(n)$ is the $i$ th sub-band symbol before filtering, $f_{i}(n)$ is the impulse response of the $i$ th sub-band filter at the beginning, and $M$ is the number of sub-band.

The transmit signal of the F-OFDM system is as follows:

$$
x(n)=\sum_{i=0}^{M-1} x_{i}(n) .
$$

The channel output signal is given by

$$
\begin{aligned}
r(n) & =x(n) * h(n)+z(n) \\
& =\sum_{i=0}^{M-1} x_{i}(n) * h(n)+z(n) \\
& =\sum_{i=0}^{M-1}\left[g_{i}(n) * f_{i}(n) * h(n)\right]+z(n) .
\end{aligned}
$$

2.2. Construction of Grid Multiscroll Chaotic System. In this paper, the new grid multiscroll chaotic system is constructed with the combination of sign functions, which act as nonlinear functions. So, the dimensionless equation of the state is constructed by the following equation:

$$
\left\{\begin{array}{l}
\frac{\mathrm{d} x}{\mathrm{~d} t}=y-a \cdot \operatorname{sgn}(y), \\
\frac{\mathrm{d} y}{\mathrm{~d} t}=z-b \cdot \operatorname{sgn}(z), \\
\frac{\mathrm{d} z}{\mathrm{~d} t}=-c x-c y-d z+e \cdot \operatorname{sgn}(x+y) .
\end{array}\right.
$$

When control parameters $a=1.4, b=1.4, c=1.5, d=0.8$, and $e=3.9$, the grid multiscroll chaotic system is in a state of chaos.

2.3. Grid Scroll Chaotic Extended Sequence Algorithm. The traditional SLM algorithm is a kind of PAPR suppression algorithm without signal distortion. However, the receiver needs to know the selected random phase sequence to demodulate, so it also needs to transmit the corresponding random phase sequence information in addition to the data information. It is restricted in practical application. In this paper, the pseudo random sequences are generated by the proposed grid multiscroll chaotic maps. The pseudo random sequences are generated by the initial value iterations. It only needs to transmit its initial value, which reduces the complexity of the operation and the side band information transmission. Aiming at the shortcomings of the traditional SLM algorithm, the grid scroll chaotic extended sequence (GSCES) algorithm is proposed in this paper. The original signals are divided into real part and imaginary part, the proposed grid multiscroll chaotic maps are used as random phase sequence vectors, the IFFT transforms are performed, respectively, the PAPR is calculated by linear superposition, and the minimum PAPR is selected for transmission as shown in Figure 2.

As shown in Figure 2, the 5G F-OFDM data block is $x(t)$, the serial input signal $x(t)$ is symbol mapped, which is divided into $2 M$ subchannels with serial-to-parallel conversion, the signal $x(t)$ is divided into the real part and the imaginary part, and the transmission is carried out on the $M$ 


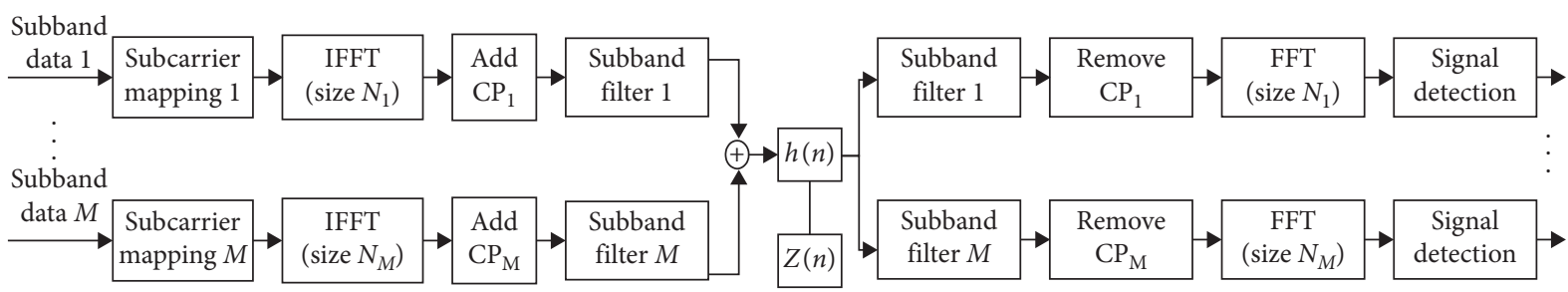

Figure 1: The downlink of F-OFDM system.

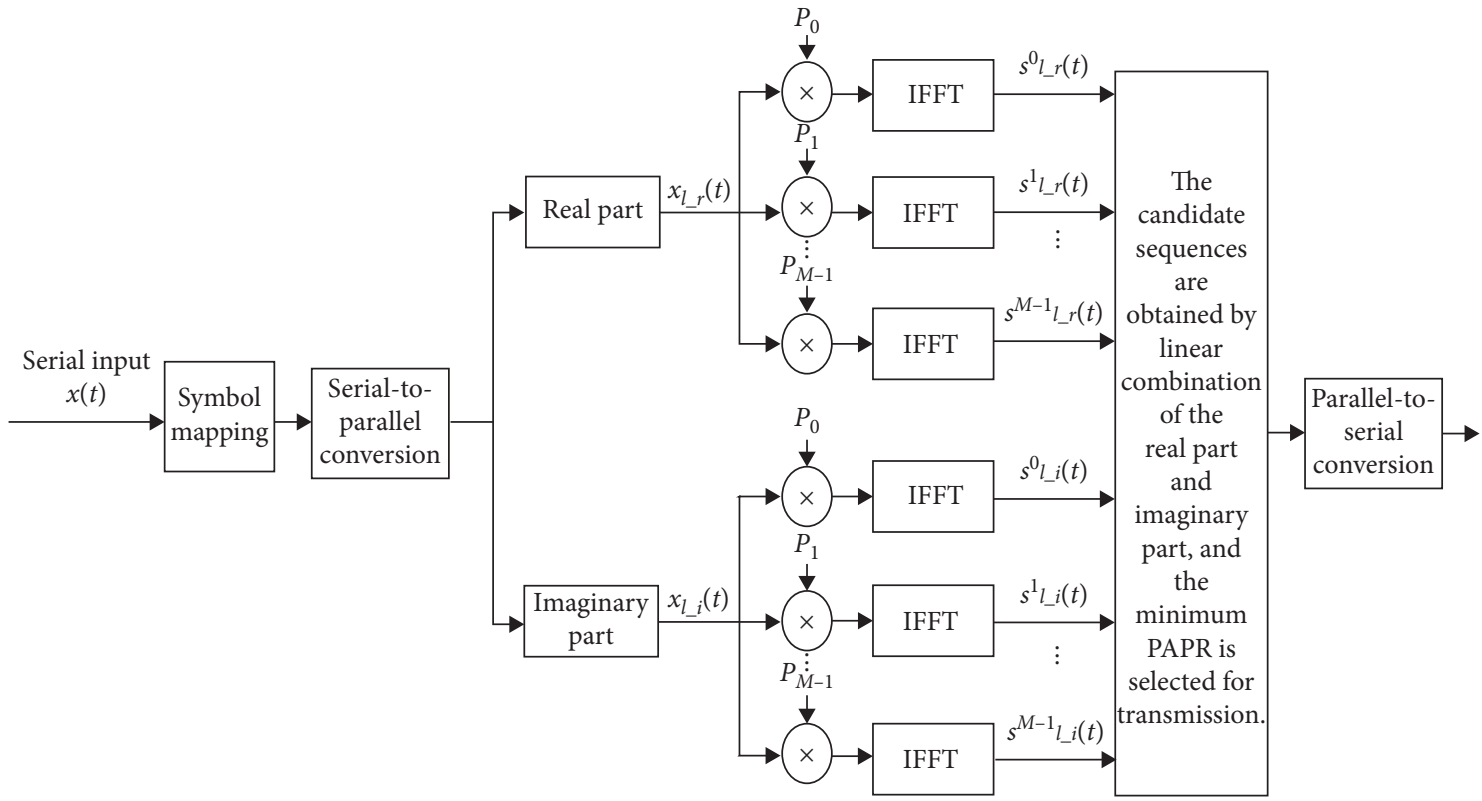

FIGURE 2: Schematic diagram of the grid scroll chaotic extended sequence algorithm.

subchannel, respectively. The proposed grid multiscroll chaotic maps are used to generate random phase sequence vector $P$ with different $N$ lengths, and then the $m$ th random phase sequence vector is $P_{m}=\left(P_{m, 0}, P_{m, 1}, \ldots, P_{m, N-1}\right)$, $0 \leq m \leq M-1$. The $l$ th data block $x_{l}(t)$ is divided into the real part $x_{l_{-} r}(t)$ and the imaginary part $x_{l_{-} i}(t)$, which are, respectively, dot produced with the $m$ th random phase sequence vector $P_{m}$, then IFFT transforms are performed, and the candidate transmission sequences $s_{l_{-r}}^{m}(t)$ and $s_{l_{-i}}^{m}(t)$ are produced:

$$
\begin{aligned}
s_{l_{-} r}^{m}(t) & =\operatorname{IFFT}\left(x_{l_{-} r}(t) \otimes P_{m}\right) \\
& =\operatorname{IFFT}\left(x_{l_{-}, 0}(t) P_{m, 0}, x_{l_{-} r, 1}(t) P_{m, 1}, \ldots, x_{l_{-}, N-1}(t) P_{m, N-1}\right), \\
s_{l_{-i}}^{m}(t) & =\operatorname{IFFT}\left(x_{l_{-} i}(t) \otimes P_{m}\right) \\
& =\operatorname{IFFT}\left(x_{l_{-}, 0}(t) P_{m, 0}, x_{l_{-} i, 1}(t) P_{m, 1}, \ldots, x_{l_{-}, N-1}(t) P_{m, N-1}\right), \\
& 0 \leq m \leq M-1,
\end{aligned}
$$

where $\otimes$ is dot product operation between two vectors, IFFT is discrete Fourier inverse transformation, $s_{l_{-} r}^{m}(t)$ is the real part of the candidate sequence, and $s_{l_{-i}}^{m}(t)$ is the imaginary part of the candidate sequence.
The candidate sequence $s_{l}^{k, q}(t)$ are obtained by linear combination of the real part $s_{l_{-} r}^{k}(t)$ and imaginary part $s_{l_{-i}}^{q}(t)$ :

$$
\begin{aligned}
s_{l}^{k, q}(t) & =\alpha_{k} s_{l_{-} r}^{k}(t) \pm j \beta_{g} s_{l_{-} i}^{q}(t) \\
& =\alpha_{k} \operatorname{IFFT}\left(x_{l_{-} r}(t) \otimes P_{k}\right) \pm j \beta_{g} \operatorname{IFFT}\left(x_{l_{-} i}(t) \otimes P_{q}\right) .
\end{aligned}
$$

If $\alpha_{k} P_{k} \pm j \beta_{g} P_{g}$ is the unit phase sequence vector, that is $\left|\alpha_{k}\right|=\left|\beta_{g}\right|=1 / \sqrt{2}$, and the positive and negative polarity have the same PAPR, then

$$
\begin{aligned}
s_{l}^{k, q}(t) & =\frac{1}{\sqrt{2}} \operatorname{IFFT}\left(x_{l_{-} r}(t) \otimes P_{k} \pm j x_{l_{-i}}(t) \otimes P_{q}\right) \\
& =\frac{1}{\sqrt{2}}\left(s_{l_{-} r}^{k}(t) \pm j s_{l_{-i}}^{q}(t)\right) .
\end{aligned}
$$

According to equation (7), the number of expanded candidate transmission sequences $s_{e l}(t)$ increases from original $M$ to $M^{2}$, and it is expressed as

$$
\begin{aligned}
& s_{e l}(t)=\left\{s_{l}^{0}(t), s_{l}^{1}(t), \ldots, s_{l}^{n}(t), \ldots, s_{l}^{M^{2}-1}(t)\right\}, \\
& 0 \leq n \leq M^{2}-1 .
\end{aligned}
$$


In the candidate transmission sequence of $l$ th data block, the minimum PAPR is selected as $D_{l}$, namely,

$$
D_{l}=\underset{0 \leq n \leq M^{2}-1}{\arg \min }\left\{\operatorname{PAPR}\left(s_{l}^{n}(t)\right)\right\}, \quad 0 \leq l \leq M-1 .
$$

So, the output sequence is as follows:

$$
D=\sum_{l=0}^{M-1} D_{l} \text {. }
$$

\section{Experiment}

According to the modular circuit design method, equation (4) has been carried out by the differential-integral transforms; we have the following equation:

$$
\left\{\begin{array}{l}
x=\int[y-a \cdot \operatorname{sgn}(y)] \mathrm{d} \tau \\
y=\int[z-b \cdot \operatorname{sgn}(z)] \mathrm{d} \tau \\
z=\int[-c x-c y-d z+e \cdot \operatorname{sgn}(x+y)] \mathrm{d} \tau .
\end{array}\right.
$$

According to equation (11), the design of the circuit is shown in Figure 3. The model of the operational amplifier is TL082. The power supply voltage of all active devices is $\pm 15 \mathrm{~V}$. All resistors are precision adjustable resistors or precision adjustable potentiometers.

\section{Results and Discussion}

4.1. Dynamics of Lyapunov Exponents. For the system corresponding equation (4), the Runge-Kutta integration method is used with the length of time $t=(0,200)$, initial values $(x, y, z)=(0.02,0.01,0.03)$, and the numerical simulation result of the Lyapunov exponents is shown in Figure 4.

The Lyapunov exponents of the proposed system are given as $\mathrm{LE}_{1}=0.055545, \mathrm{LE}_{2}=0.025606$, and $\mathrm{LE}_{3}=-0.881151$, also $\mathrm{LE}_{1}+\mathrm{LE}_{2}+\mathrm{LE}_{3}=-0.8$. Moreover, the fractal dimension is given by

$$
\begin{aligned}
\mathrm{LD} & =j+\frac{1}{\left|\mathrm{LE}_{j+1}\right|} \sum_{i=1}^{j} \mathrm{LE}_{i}=2+\frac{0.055545+0.025606}{0.881151} \\
& =2.0921 .
\end{aligned}
$$

The proposed system has positive Lyapunov exponent and the dimension of Lyapunov is fractional, which further confirms that the system is in the state of chaos and the calculated Lyapunov exponent is reasonable. Moreover, this system produces strange attractor dimension $\mathrm{LD}=2.0921$, in line with the geometric characteristics of the chaotic attractor.

4.2. Poincare Map of Grid Multiscroll Chaotic System. For the system corresponding equation (4), the RungeKutta integration method is used with the length of time $t=(0,2600)$ and initial values $(x, y, z)=(0.02,0.01,0.03)$. The
Poincare map of the grid multiscroll chaotic system is shown in Figure 5.

A Poincare section is selected in the phase space, which is used to cut off all the motion trajectories for observing section cut point. From Figure 5, we can see that the Poincare map of the grid multiscroll chaotic system is a piece of dense point set and has a hierarchical structure, which verifies the system's chaotic motion state.

4.3. Hardware Results. The hardware circuits shown in Figure 3 can be obtained, and experimental results are shown in Figure 6.

From Figure 6, it can be seen that the hardware circuit experiment results of the analog oscilloscope generate $2 \times 2 \times 2$-grid multiscroll chaotic attractors, which verifies the physical feasibility of the proposed system. The phase rails of the proposed grid multiscroll chaotic attractor are separated in more directions and have more complex dynamic characteristics. Therefore, the grid multiscroll chaotic map is used as random phase sequence vectors. Compared with the traditional pseudo random sequences, the proposed grid multiscroll chaotic sequence can provide a large number of random sequences with good correlation characteristics, random characteristics, and high security, which meet the requirement of $5 \mathrm{G}$ mass connection.

4.4. PAPR Performance Simulation Results. In this paper, the QPSK modulation is adopted, the subcarrier number equals to 128 , and the complementary cumulative distribution function (CCDF) is used to describe the PAPR distribution of the proposed system. In paper [6], the selected data utilization (SDU) algorithm is proposed to rotate the phase factor with specific selection conditions, which improves the PAPR performance of the traditional SLM algorithm. The PAPR performance comparison between the proposed GSCES algorithm and the traditional SLM algorithm and the SDU algorithm is shown in Figure 7.

In Figure 7, the variable original is the PAPR value calculated by $1 \times 128$ original signal.

In the traditional SLM algorithm, the $1 \times 128$ original signal is input data, $L$ groups input data are copied and are dot product of random phase vectors, IFFT transforms are calculated, and the PAPR values are obtained, and the variable SLM is the minimum PAPR value of the $L$ candidate sequences. The traditional SLM algorithm using random phase vectors requires the transmission of phase information of subchannel, so the side band information is very huge, which not only increases the complexity but also reduces the efficiency of the system.

In the proposed GSCES algorithm, the $1 \times 128$ original signal is the input data, $L$ groups input data are copied, the real part and the imaginary part of input data are separated, $L$ random phase vectors are generated by the proposed grid multiscroll chaotic maps, the real part and the imaginary part are separately dot product with the random phase vectors, IFFT transforms are calculated, and real and imaginary data are linearly added. The proposed GSCES algorithm extends the candidate sequence to $L^{2}$ groups, and 


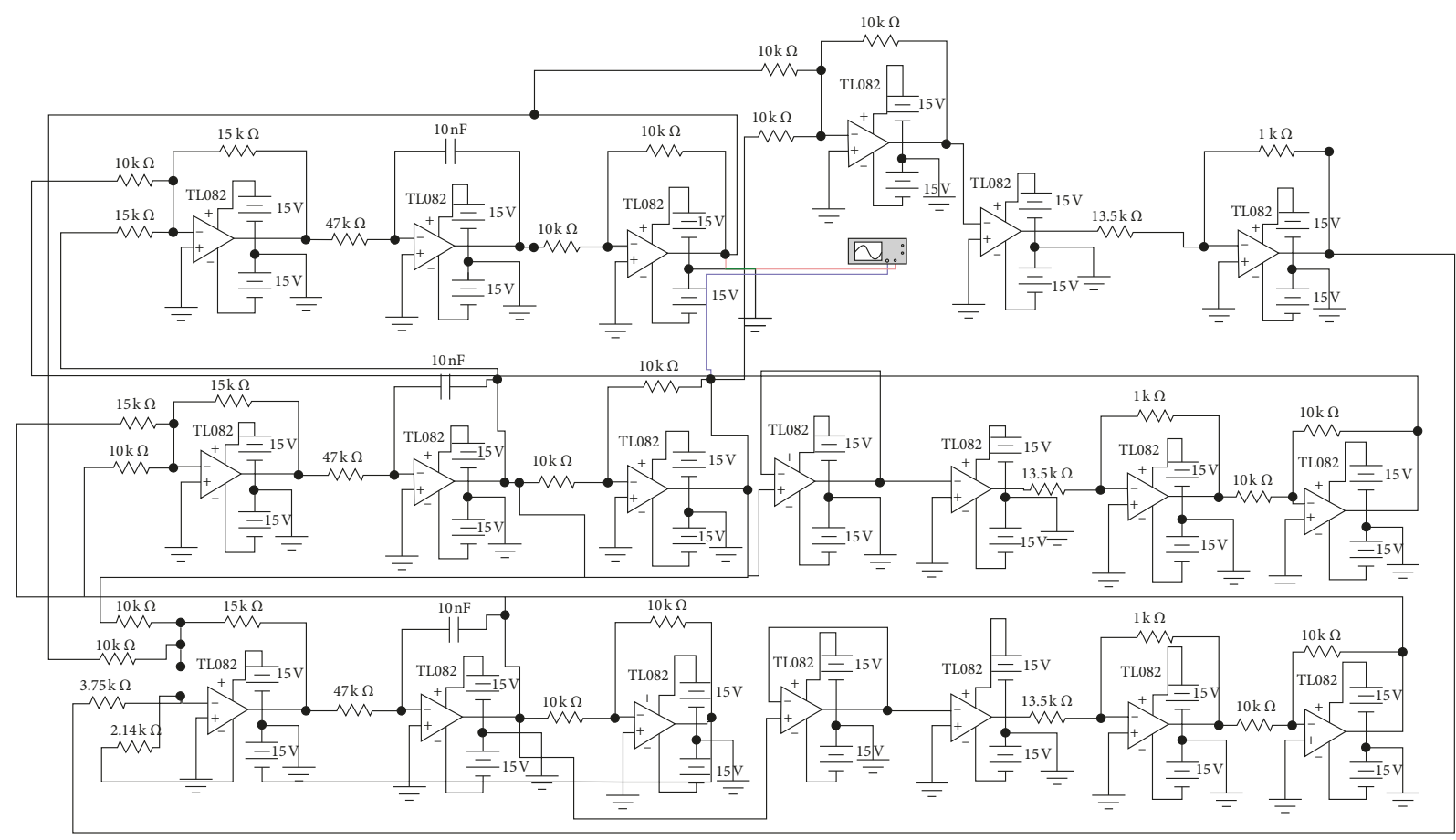

FIgURE 3: Hardware circuit diagram.

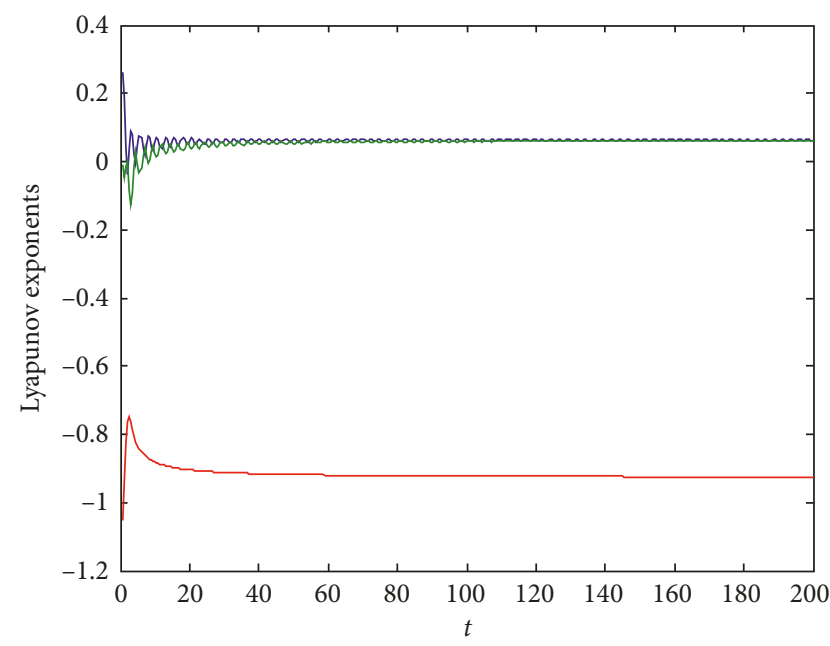

Figure 4: Lyapunov exponents of the proposed system.

variable LSLM is the minimum PAPR value of $L^{2}$ candidate sequences.

In Figure 7, compared with the traditional SLM algorithm and SDU algorithm, the proposed GSCES algorithm effectively reduces the PAPR of the 5G F-OFDM system, increases the candidate sequences, reduces calculation, and transmits data more efficiently.

4.5. BER Performance Simulation Results. The F-OFDM signal divides the frequency band of the system into several different sub-bands, which can be configured with different link parameters according to the actual service requirements, such as different subcarrier spacing, IFFT/FFT size, and CP

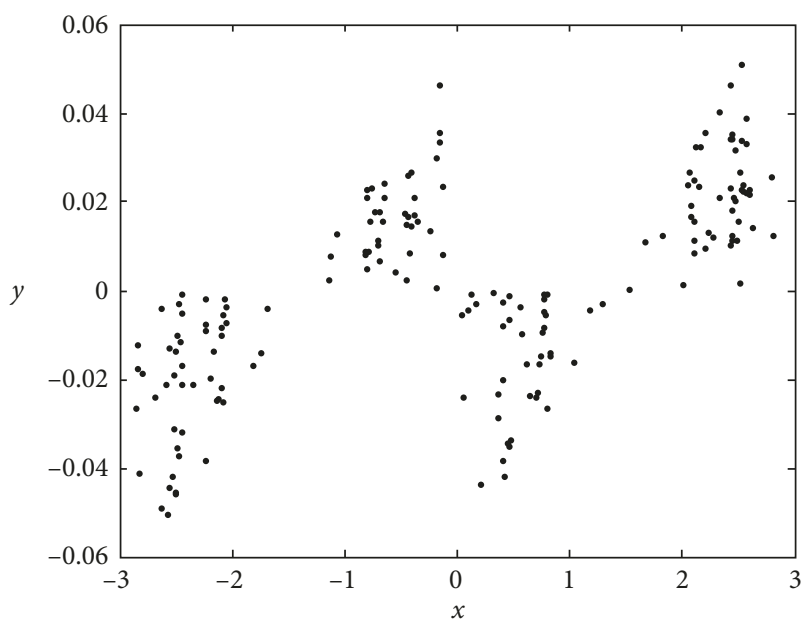

Figure 5: Poincare map of the grid multiscroll chaotic system.

length. In this section, we evaluate the bit error rate (BER) performance of the proposed GSCES algorithm in the F-OFDM system. In the additive white Gaussian noise (AWGN) channel, the system performance test is carried out, and the specific simulation parameter configuration of the F-OFDM system is shown in Table 1.

The configuration of link parameters in this paper is shown in Table 1. In the F-OFDM system, the BER performance of the proposed GSCES algorithm is shown in Figure 8.

From Figure 8, it can be seen that the BER performance of the proposed GSCES algorithm has a certain degree of degradation compared with the original signal, but the degree of degradation is not large. Combining with Figure 7, 


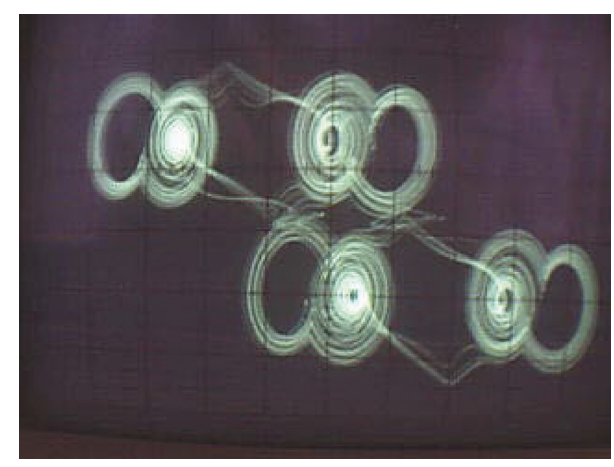

Figure 6: Hardware circuit experimental results.

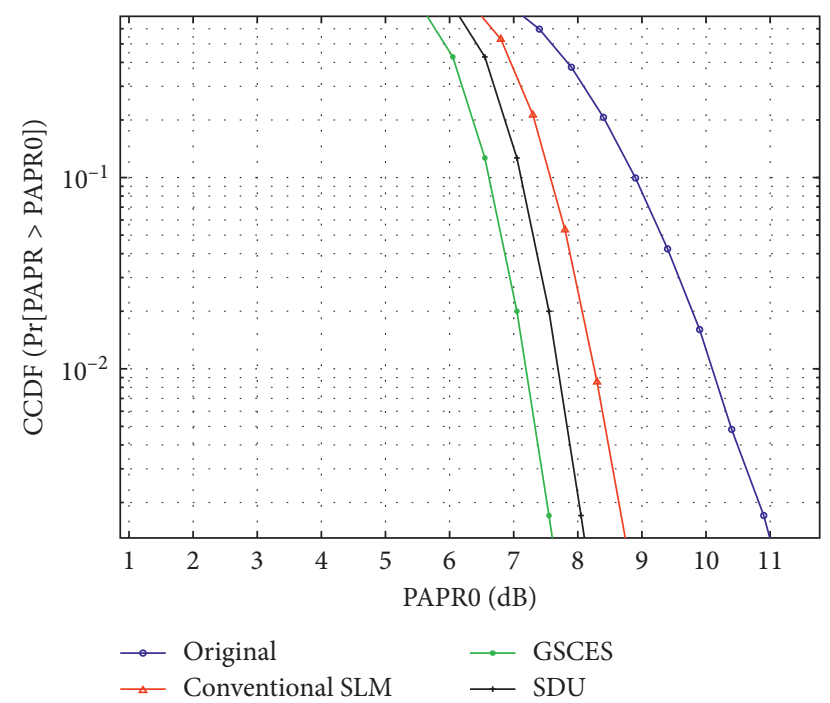

FIGURE 7: The PAPR performance comparison results.

TABLE 1: Simulation setup.

\begin{tabular}{lc}
\hline Parameter & Value/description \\
\hline Subcarrier size & 64 \\
IFFT/FFT size & 1024 \\
Baseband modulation mode & QPSK \\
Subcarrier spacing & $15 \mathrm{KHz}$ \\
CP length & 16 \\
Symbol duration & $66.7 \mu \mathrm{s}$ \\
Channel model & AWGN \\
Type of filters & Square root raised cosine \\
\hline
\end{tabular}

it can be seen that the proposed GSCES algorithm can effectively improve the PAPR performance of the system, and when the transmission signal meets certain signal-to-noise ratio (SNR) conditions, it will not affect the BER performance of the system.

4.6. Computational Complexity Analysis. Assuming that both the SLM algorithm and the proposed GSCES algorithm generate $L^{2}$ candidate sequences, the number of subcarriers is $R$, the oversampling rate is $Q$, and the IFFT transformation of $R Q$ points requires $(R Q / 2) \log _{2} R Q$ complex

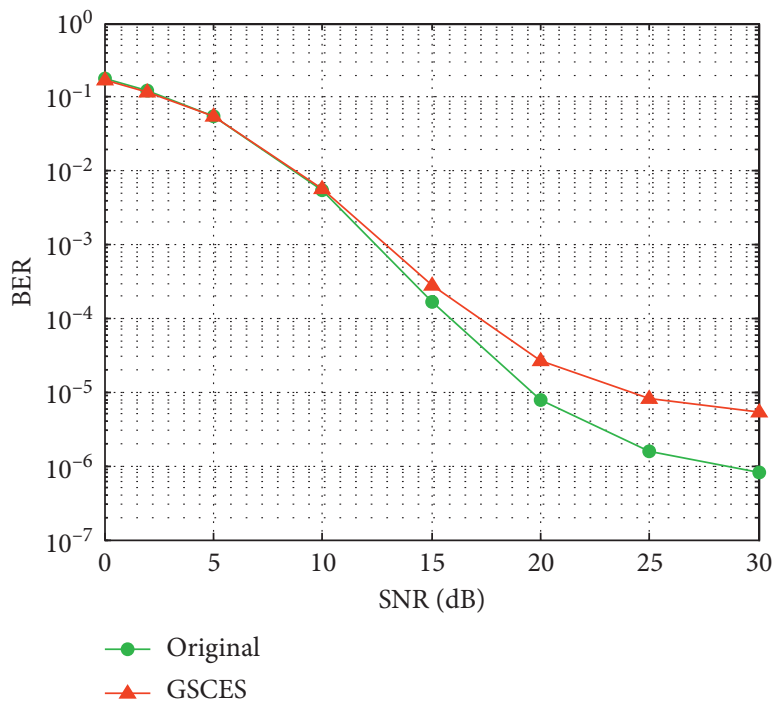

FIgURE 8: The BER performance results.

TABle 2: Computational complexity comparison of different algorithms.

\begin{tabular}{lcc}
\hline Algorithm & $\begin{array}{c}\text { Number of complex } \\
\text { multiplications }\end{array}$ & $\begin{array}{c}\text { Number of complex } \\
\text { additions }\end{array}$ \\
\hline SLM & $\left(L^{2} R Q / 2\right) \log _{2} R Q$ & $L^{2} R Q \log _{2} R Q$ \\
GSCES & $(L R Q / 2) \log _{2} R Q$ & $L R Q \log _{2} R Q+L^{2} R Q$ \\
GSCES/SLM & $1 / L$ & $(1 / L)+\left(1 / \log _{2} R Q\right)$ \\
\hline
\end{tabular}

multiplications and $R Q \log _{2} R Q$ complex additions. The SLM algorithm needs $L^{2}$ IFFT transforms to generate $L^{2}$ phase sequences, i.e., a total of $\left(L^{2} R Q / 2\right) \log _{2} R Q$ complex multiplications and $L^{2} R Q \log _{2} R Q$ complex additions. For the proposed GSCES algorithm, the Lth IFFT transform generates the real and imaginary parts of $L$ candidate sequences.

That is to say, $(L R Q / 2) \log _{2} R Q$ complex multiplications and $L R Q \log _{2} R Q$ complex additions are needed. Then, the linear combination of the real and imaginary parts of $L$ candidate sequences is used to generate $L^{2}$ candidate sequences, which requires $L^{2} R Q$ complex additions.

When the number of the candidate sequence equals to $L^{2}$, the computational complexity of the proposed GSCES algorithm is compared with that of the SLM algorithm as shown in Table 2.

Table 2 shows that when the numbers of candidate sequences are all $L^{2}$, the number of complex multiplication needed by the proposed GSCES algorithm is $1 / L$ of that of the SLM algorithm and the number of complex addition is $\left((1 / L)+\left(1 / \log _{2} R Q\right)\right)$ of the SLM algorithm. With the increase of the number of candidate sequences, the ratio decreases continuously. So, the proposed GSCES algorithm expands the number of candidate sequences by using the segmentation method and linear combination transformation. Compared with the SLM algorithm, the computational complexity is greatly reduced and the data can be transmitted more efficiently. 


\section{Conclusions}

Aiming at the shortcomings of the traditional SLM algorithm, the GSCES algorithm is proposed to solve the high PAPR problem of 5G F-OFDM systems. The proposed grid multiscroll chaotic maps instead of the traditional pseudo random sequence are used as random phase sequence vectors, which provide a large number of random sequences with good correlation characteristics, random characteristics, and high security. The proposed random phase sequence vectors can meet the needs of $5 \mathrm{G}$ mass connections. The original input signals are divided into real part and imaginary part, which are, respectively, multiplied by the proposed grid multiscroll chaotic sequences. The IFFT transforms are performed, respectively, the PAPR values are calculated by linear superposition, and the minimum PAPR value is selected for transmission. The numerical simulation results show that the proposed GSCES algorithm effectively increases the numbers of candidate sequences, reduces the PAPR values, the redundant information transmission, and the side band information transmission, and improves the transmission efficiency and security of the 5G F-OFDM system. Moreover, the hardware experimental results produce at $2 \times 2 \times 2$-grid multiscroll chaotic attractor, which further proves the physical realization of the proposed system. The proposed schemes have a broad application prospect in 5G multicarrier modulation technology.

\section{Abbreviations}

PAPR: Peak-to-average power ratio

F-OFDM: Filtered-orthogonal frequency division multiplexing

SLM: $\quad$ Selective mapping

GSCES: Grid scroll chaotic extended sequence

SI: $\quad$ Side information

CP: $\quad$ Cycle prefix

CCDF: Cumulative distribution function

BER: $\quad$ Bit error rate

AWGN: Additive white Gaussian noise

SNR: $\quad$ Signal-to-noise ratio

SDU: $\quad$ Selected data utilization.

\section{Data Availability}

The data used to support the findings of this study are included within the article.

\section{Conflicts of Interest}

The authors declare that they have no conflicts of interest.

\section{Authors' Contributions}

Yingjie Ma wrote the majority of the text and performed the design and implementation of the algorithm. Ya Li performed the design and implementation of the hardware circuit. Geng Zhao performed the numerical simulation experiment. Ping Zeng and Yatao Yang contributed text to earlier versions of the manuscript and commented on and approved the manuscript. All authors read and approved the final manuscript.

\section{Acknowledgments}

This work is supported by the National Natural Science Foundation of China (no. 61772047), Science and Technology Program of Guangzhou, China (201904010302), and Doctoral Scientific Research Startup Fund of Guangdong Polytechnic Normal University (991512204).

\section{References}

[1] C. Jayawickrama, S. Kumar, and H. Song, "Novel wideband chaotic approach LNA with microcontroller compatibility for 5G wireless secure communication," Microwave and Optical Technology Letters, vol. 60, no. 2, pp. 488-494, 2018.

[2] G. U. Maheswari, A. Govindasamy, and S. J. Thiruvengadam, "Performance analysis of filter bank multicarrier system with non-linear high power amplifiers for $5 \mathrm{G}$ wireless networks," IET Signal Processing, vol. 11, no. 1, pp. 66-72, 2017.

[3] N.-T. Le, D. Jayalath, and J. Coetzee, "Spectral-efficient resource allocation for mixed services in OFDMA-based $5 \mathrm{G}$ heterogeneous networks," Transactions on Emerging Telecommunications Technologies, vol. 29, no. 1, p. e3267, 2018.

[4] A. Kheli, L. Talbi, D. Slimani, and J. LeBel, "MRC SC-FDMA scheme performance evaluation based on measurements at 30 $\mathrm{GHz}$ for 5G communications," Physical Communication, vol. 25, pp. 462-468, 2017.

[5] S. Wang, J. S. Thompson, and P. M. Grant, "Closed-form expressions for ICI/ISI in filtered OFDM systems for asynchronous 5G uplink," IEEE Transactions on Communications, vol. 65, no. 11, pp. 4886-4898, 2017.

[6] P. D. Pamungkasari and Y. Sanada, "Shift estimation with delayed correlation cyclic-selective mapping (Cyclic-SLM) PAPR reduction in OFDM system," Wireless Personal Communications, vol. 91, no. 2, pp. 739-753, 2016.

[7] Y. Xiao, Z. Wang, J. Cao et al., "Time-frequency domain encryption with SLM scheme for physical-layer security in an OFDM-PON system," Journal of Optical Communications and Networking, vol. 10, no. 1, pp. 46-51, 2018.

[8] W.-W. Hu, "SLM-based ACO-OFDM VLC system with lowcomplexity minimum amplitude difference decoder," Electronics Letters, vol. 54, no. 3, pp. 144-146, 2018.

[9] S. Vaiyamalai, S. Mahesula, and S. K. S. Dhamodharan, "PAPR reduction in SLM-OFDM system using lehmer sequence without explicit side information," Wireless Personal Communications, vol. 97, no. 4, pp. 5527-5542, 2017.

[10] S. A. Adegbite, S. McMeekin, and B. G. Stewart, "Computational efficient SLM-OFDM receiver for time-invariant indoor fading channel," Wireless Personal Communications, vol. 97, no. 1, pp. 661-674, 2017.

[11] V. Sudha, M. Syamkumar, and D. S. Kumar, "A low complexity modified SLM and companding based PAPR reduction in localized OFDMA," Wireless Personal Communications, vol. 96, no. 2, pp. 3207-3226, 2017.

[12] J.-g. Yuan, Q. Shen, J.-x. Wang, Y. Wang, J.-Z. Lin, and Y. Pang, "A novel improved SLM scheme of the PAPR reduction technology in CO-OFDM systems," Optoelectronics Letters, vol. 13, no. 2, pp. 138-142, 2017.

[13] M. Fallahzadeh and M. Ferdosizadeh, "Blind SLM for PAPR reduction of Alamouti DSFBC systems," IET Communications, vol. 11, no. 3, pp. 451-457, 2017. 


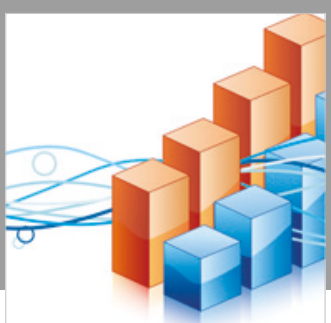

Advances in

Operations Research

\section{-n-m}
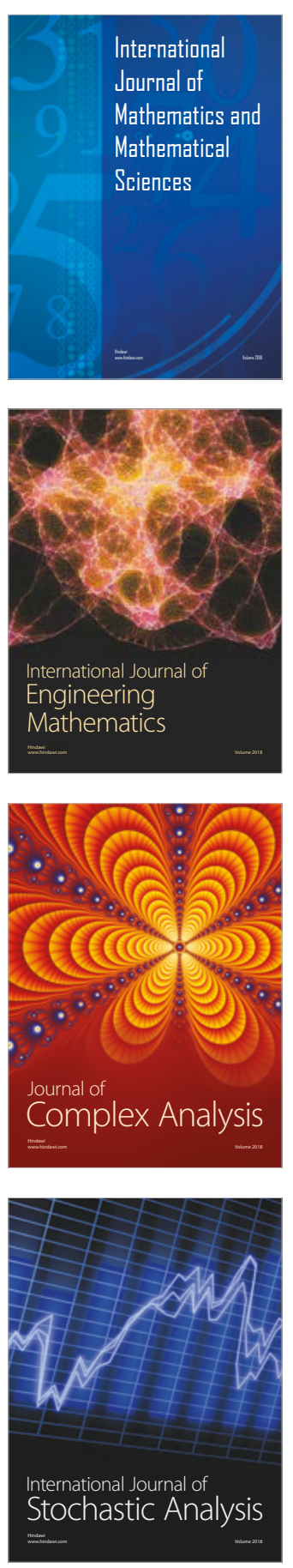
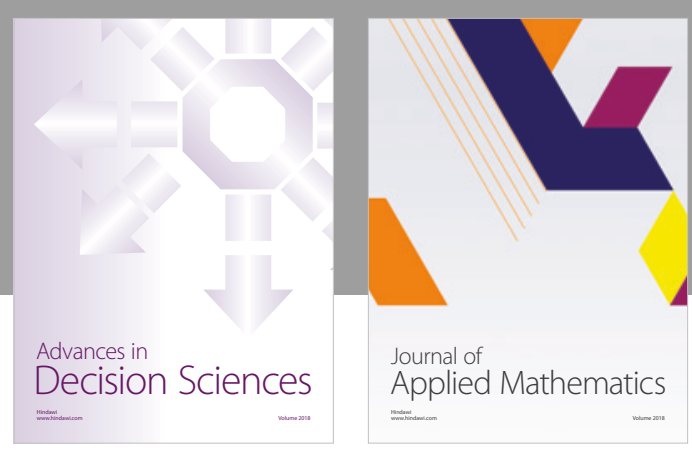

Journal of

Applied Mathematics
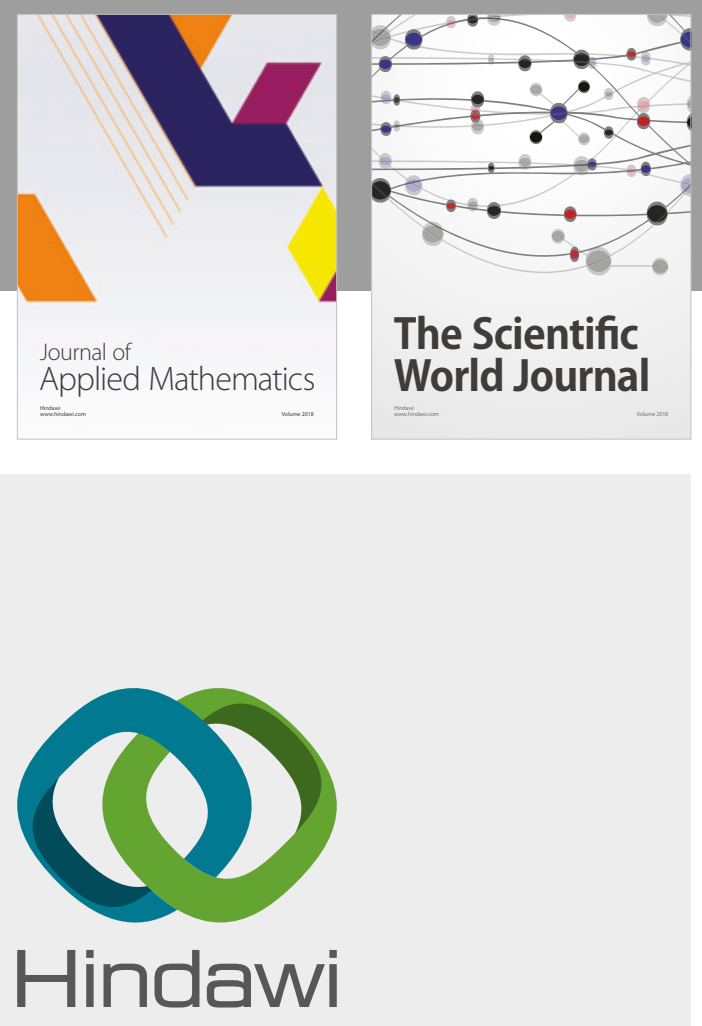

Submit your manuscripts at

www.hindawi.com

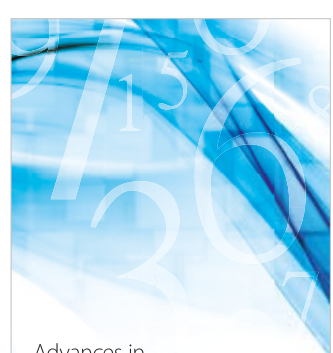

Advances in
Numerical Analysis
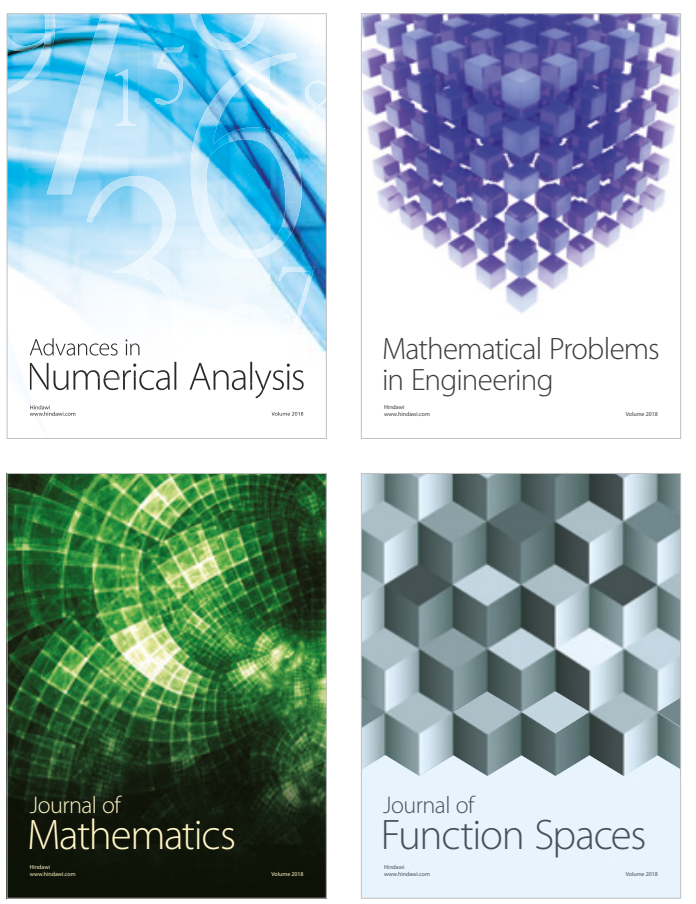

Mathematical Problems in Engineering

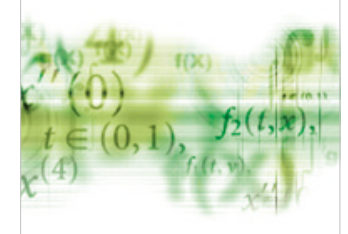

International Journal of

Differential Equations

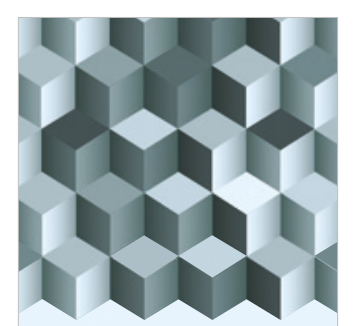

Journal of

Function Spaces
The Scientific

World Journal

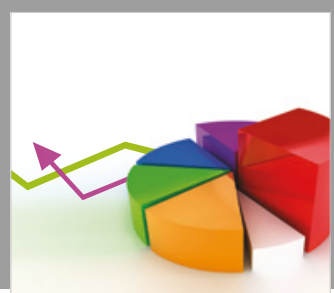

Journal of

Probability and Statistics
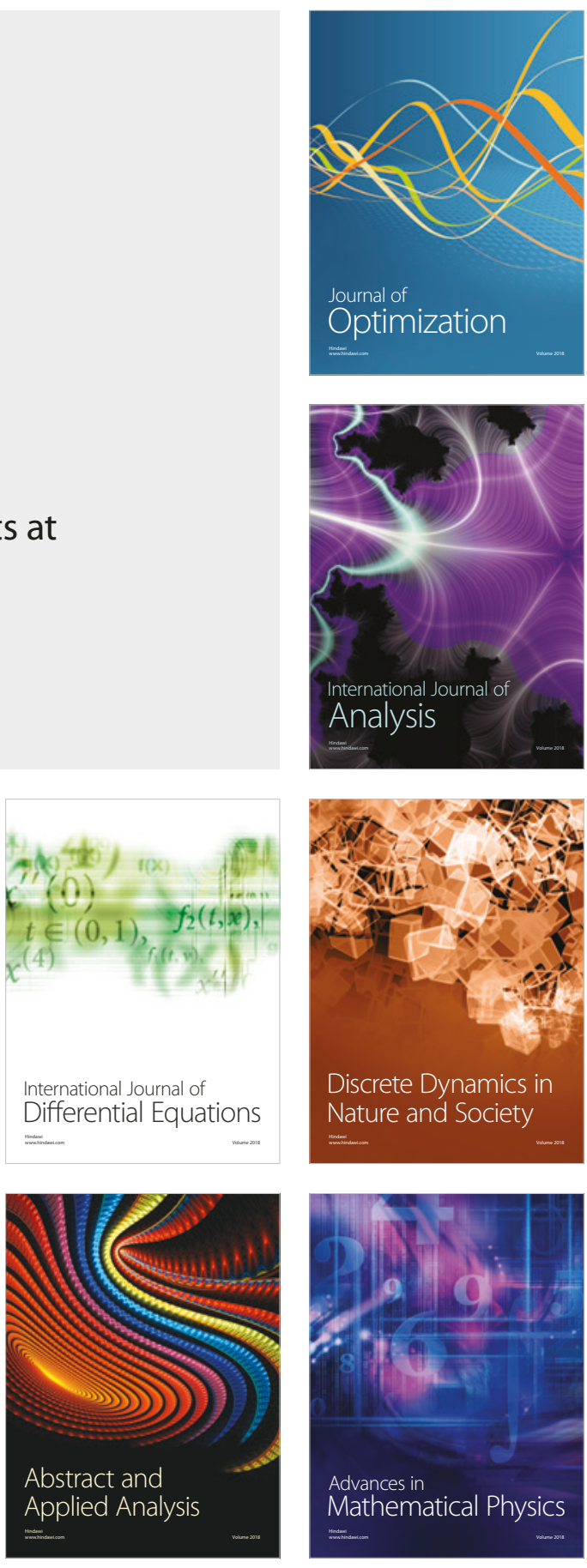